\title{
Visceral Adipose Tissue Volume Estimated at Imaging Sites 5-6 cm Above L4-L5 Is Optimal for Predicting Cardiovascular Risk Factors in Obese Japanese Men
}

\author{
Rina So, ${ }^{1,2}$ Hiroyuki Sasai, ${ }^{3,4}$ Tomoaki Matsuo, ${ }^{1}$ Takehiko Tsujimoto, ${ }^{1}$ Miki Eto, ${ }^{5}$ \\ Kousaku Saotome ${ }^{6}$ and Kiyoji Tanaka ${ }^{5}$ \\ ${ }^{1}$ Graduate School of Comprehensive Human Sciences, University of Tsukuba, Tsukuba, Japan \\ ${ }^{2}$ JSPS Research Fellow of the Japan Society for the Promotion of Science, Tokyo, Japan \\ ${ }^{3}$ National Institutes of Health, Bethesda, USA \\ ${ }^{4}$ JSPS Research Fellow in Biomedical and Behavioral Research at NIH, Bethesda, USA \\ ${ }^{5}$ Faculty of Health and Sport Sciences, University of Tsukuba, Tsukuba, Japan \\ ${ }^{6}$ Center for Cybernics Research, University of Tsukuba, Tsukuba, Japan
}

\begin{abstract}
The association between visceral adipose tissue (VAT) with cardiovascular disease (CVD) has been clearly demonstrated. Although typical VAT area at 4th and 5th lumbar vertebrae (L4-L5) is used to approximate VAT volume, growing evidence has suggested that this measurement site may not be ideal. However, these findings for Asian people remain unclear. Thus, we searched for the better VAT measurement sites associated with CVD risk factors in obese, Japanese men. Eighty-two obese men were included in a cross-sectional study. Among these participants, 37 men completed the 12-week intervention (90 min and $3 \mathrm{~d} /$ week) were used for addressing longitudinal association between the VAT measurement sites and CVD risk factors. Consecutive MRI images (from $3 \mathrm{~cm}$ below L4-L5 to $20 \mathrm{~cm}$ above L4-L5) were used to explore the relationship between each VAT area and CVD risk factors (total cholesterol, HDL cholesterol, triglycerides, glucose, insulin and blood pressure). The images located only 5-9 cm above L4-L5 had significant correlations with HDL cholesterol and triglycerides, but L4-L5 site did not in the cross-sectional analysis. In response to exercise, the image located $5 \mathrm{~cm}$ above L4-L5 showed the highest correlations with changes in total cholesterol $(r=0.46)$ and glucose $(r=0.36)$. Also, the image located $6 \mathrm{~cm}$ above L4-L5 showed highest correlations with changes in triglycerides $(r=0.37)$ and insulin $(r=0.37)$. Thus, the range of VAT images located 5-6 cm above L4-L5 may be optimal for identifying CVD risk factors compared to a typical site of L4-L5.
\end{abstract}

Keywords: cardiovascular disease; exercise; measurement site; multiple-slice image; visceral adipose tissue Tohoku J. Exp. Med., 2012, 227 (4), 297-305. (C) 2012 Tohoku University Medical Press

Visceral adipose tissue (VAT) has emerged as a powerful predictor for obesity and is closely associated with cardiovascular disease (CVD). Specifically, the accumulation of abdominal VAT seems to be a strong predictor of dyslipidemia (Nieves et al. 2003), impaired glucose tolerance (Janssen et al. 2002), insulin resistance (Ross et al. 2002), and the incidence of hypertension (Yamazaki and Hayashi 2002). These observations emphasize the notion that VAT should be the primary target for strategies designed to reduce obesity and related CVD risk factors. Although exercise and diet together can improve the loss of weight, VAT and abdominal circumference in obese humans, exercise seems to be the more efficient method for promoting weight and VAT loss (Physical Activity Guidelines Advisory Committee Report. 2009), and physical activity by itself could lead to moderate body weight loss (Jakicic 2003; Burke and Wang 2011; Straznicky et al. 2011). Thus, by reducing VAT and CVD risk with exercise we may be able to limit CVD, and this should be considered the endpoint for obesity treatment. Even though there are several methods of evaluating VAT, it is generally accepted that computed tomography (CT) and magnetic resonance imaging (MRI) are the in vivo methods of choice for directly assessing the VAT content of human subjects. Although there are several protocols used for quantifying VAT, as a compromise between accuracy and cost, previous epidemiologic and interventional studies have commonly used single-slice images at fourth lumbar (L4) and fifth lumbar (L5) intervertebral space as a representative measure of the VAT (Tanaka et al. 2004; Fox et al. 2007; Rosito et al. 2008;

Received May 8, 2012; revision accepted for publication June 28, 2012. doi: 10.1620/tjem.227.297

Correspondence: Rina So, MS., Graduate School of Comprehensive Human Science, University of Tsukuba, 1-1-1 Tennodai, Tsukuba, Ibaraki 305-8577, Japan.

e-mail: rina@stat.taiiku.tsukuba.ac.jp 
Sasai et al. 2008). However, recent studies of Caucasian (Kuk et al. 2006), White and African-American cohorts (Demerath et al. 2007b) have indicated that the commonly used L4-L5 image site may not be the best location to estimate the VAT volume, whereas the findings for Asian people remain unclear. Furthermore, it is suggested that L4-L5 may not be an appropriate site for investigating the association between VAT and CVD risk factors (Kvist et al. 1988; Abate et al. 1997; Kuk et al. 2006; Demerath et al. 2007a; Shen et al. 2007; Irlbeck et al. 2010).

Although most previous studies are cross-sectional, Kuk et al. (2010) were the first to longitudinally examine the influence of measurement site on the prediction of VAT volume in response to exercise interventions in obese postmenopausal Caucasian women. In their study, these authors investigated an association between metabolic syndrome (MetS), a cluster of CVD risk factors, and the measurement sites for VAT volume. However, the effects on individual CVD risk factors, including total cholesterol (TC), HDLcholesterol (HDL-C), triglycerides (TG), glucose, insulin and blood pressure have not been fully investigated. To our knowledge, no previous longitudinal studies have investigated associations between the VAT measurement sites and individual CVD risk factors.

In our study, we tested whether regular exercise can alter the optimum anatomic site for measuring VAT and for predicting reductions in the VAT volume and CVD risk factors. Because previous studies had very few to no individuals of Asian descent, further attention should be given to a longitudinal study of Asians. Therefore, the aims of this study are twofold: 1) to examine the influence of singleslice image measurement sites on the VAT volume and changes in VAT volume, and 2) to determine whether the measurement site significantly affects the correlation between the single-slice image of the VAT area and CVD risk factors in response to exercise in obese, Japanese men.

\section{Methods}

\section{Participants}

We initially recruited 84 participants for a weight loss study based on exercise and/or an energy-restricted diet through advertisements in local newspapers and the distribution of flyers. Of these 84 participants, we excluded 2 men because we were unable to adequately measure their MRI images. The remaining 82 participants were included the cross-sectional study. Of these 82 participants, 37 completed the exercise program and were included in the longitudinal study. We adopted the following criteria: male, age between 30 and 60 years, and a BMI of greater than $25 \mathrm{~kg} / \mathrm{m}^{2}$ according to the domestic obesity guidelines (The Examination Committee of Criteria for 'Obesity Disease' in Japan. 2002). We excluded participants who had concomitant renal, hepatic, or cardiac diseases that could affect the outcome measures of the study. All of the participants were included in the cross-sectional analysis looking at associations between the VAT measurement areas and CVD risk factors at baseline. Of the 82 overweight and obese male subjects, 52 men who had no exercise habits (defined as continual exercise for at least 30 minutes per day 2 days per week over a year or more (Ministry of Health, Labour and
Welfare 2009)) participated in a 12 -week exercise intervention. Nine participants dropped out due to injury (1 study related and 1 nonstudy related) and scheduling conflicts. Six participants with incomplete data were also excluded from the analysis. Finally, 37 men were included in the longitudinal analysis. We fully explained the purpose and design of the study to each participant before they gave written informed consent. This study conformed to the principles outlined in the Helsinki Declaration and was approved by the Institutional Ethical Review Board.

\section{Measurement Procedures}

Anthropometry, maximal oxygen uptake, MRI, and CVD risk factors were measured at baseline for all 82 participants. The same measurements were repeated in the same order on the 37 participants who completed the exercise intervention. Energy intake and expenditure were also measured on the same participants during the intervention period. We instructed the participants not to participate in vigorous physical activity or to consume alcohol within $24 \mathrm{~h}$ prior to the measurements.

\section{Anthropometry}

Body weight was measured to the nearest $0.1 \mathrm{~kg}$ using a digital scale (WB-150; Tanita, Tokyo, Japan), and height was measured once to the nearest $0.1 \mathrm{~cm}$ using a wall-mounted stadiometer (YG-200; Yagami, Nagoya, Japan). Body mass index (BMI) was calculated as the weight (in kilograms) divided by height (in meters) squared. The abdominal circumference (The national Institute of Health and Nutrition Survey in Japan. 2008) was measured in the standing position directly on the skin surface at the level of the umbilicus. The abdominal circumference measurements were taken in duplicate to the nearest $0.1 \mathrm{~cm}$ with the mean value used for the analysis.

\section{Maximal oxygen uptake}

Maximal oxygen uptake $\left(\dot{\mathrm{V}}_{2} \max \right)$ for each participant was determined by a graded exercise test using a cycling ergometer $(828 \mathrm{E}$, Monark, Stockholm, Sweden). Following a 2-min warm-up at 30 watts, the workload increased every minute by 15 watts until volitional exhaustion. The highest oxygen uptake achieved over $30 \mathrm{~s}$ was analyzed in accordance with published criteria (Tanaka et al. 1990). If the participants did not meet these criteria, we used a workload of 15, as determined on a Rating of Perceived Exertion scale (RPE), during the test to estimate the $\dot{\mathrm{V}} \mathrm{O}_{2} \max$ (Okura and Tanaka 2000). We recorded the RPE during the last 15 seconds of each stage. We previously reported on a validated equation for the RPE method ( $n=83, r$ $=0.83$, standard error of the estimate $=3.66 \mathrm{ml} / \mathrm{kg} / \mathrm{min}$ ) (Okura and Tanaka 2000).

\section{Magnetic resonance imaging}

We refrain from eating or drinking for 2 hour prior to the measurement. Abdominal multiple-slice MRI scans were performed with a 1.5-T system (Siemens Magnetom Avanto syngo MR B15, Siemens, Erlangen, Germany). All participants received T1-weighted image scans using a body coil. The imaging parameters included a spinecho sequence with a 379-ms repetition time, an 11-ms echo time, a 48 -cm field of view and a $256 \times 256$ matrix. The participants were instructed to lie in the magnet in a supine position with the arms extended above the head. The slice thickness was $10 \mathrm{~mm}$, and the images were obtained from the ninth thoracic vertebra (T9) to the first sacral vertebra (S1) (Keith 1999). Image locations were defined rela- 
tive to the common anatomical landmark of the L4-L5 intervertebral space. The average total scanning time was 15 minutes for each participant, and all scanning was performed by the same radiological instrument technician. To compare the individual image data precisely, we limited our analyses to image sets having no missing values for any of the participants. This protocol is generally consistent with those used in other studies (Demerath et al. 2007b, 2008). The images were retrieved from the scanner according to a DICOM (Digital Imaging and Communications in Medicine) protocol. After image acquisition, the same experienced technician performed the segmentation and quantification of the subcutaneous adipose tissue and VAT for all subjects using image analysis software (SliceOmatic, Tomovision Inc, Montreal, Canada). The VAT was defined as the adipose tissue in the region enclosed by the inner aspect of the abdominal wall and the anterior margin of the vertebral body including the intra-abdominal cavity. We used a region-based thresholding method (that is, a 'region growing' function) to segment the fat regions. This method is based on the property of our acquired MRI images in which the signal intensities of fat pixels on T1-weighted images are higher than those of muscle and visceral organ pixels. The technical errors for 2 repeated readings of the same scan by the same observer for SAT and VAT volumes in our laboratory were $1.23 \%$ and $2.27 \%$, respectively. The model and method employed to segment the various tissues is fully described and illustrated elsewhere (Shen and Chen 2008).

\section{Cardiovascular disease risk factors}

A blood sample was drawn from each participant after an overnight ( $\geq 8 \mathrm{~h}$ ) fast. We detected the TC and TG levels with an enzymatic method using Determiner L TC II and Determiner L TG II, respectively (Kyowa Medex Co., Ltd, Tokyo, Japan for both). We measured the serum HDL-C levels by the heparin-manganese precipitation method using Determiner L HDL-C (Kyowa Medex Co., Ltd, Tokyo, Japan). Glucose was assayed with the Hexokinase-G-6-PDH method using L-Type Glu 2 (Wako Pure Chemical Industries, Ltd, Osaka, Japan). Insulin was measured by electrochemiluminescence immunoassay using Elecsys Insulin (Roche Diagnostics, Ltd, Tokyo, Japan) The inter- and intra-assay coefficients of variation were 0.5 $0.9 \%$ for TC, $0.4-2.2 \%$ for TG, $0.5-0.9 \%$ for HDL-C, $0.5-0.8 \%$ for glucose and $0.8-1.0 \%$ for insulin. Trained nurses measured the systolic and diastolic blood pressures using a standard mercury sphygmomanometer (cuff size: $14 \mathrm{~cm} \times 47 \mathrm{~cm}$ ) on the right arm of seated participants who had rested for at least $10 \mathrm{~min}$.

\section{Activity energy expenditure and energy intake}

The total energy expenditure, physical activity energy expenditure and number of steps taken were assessed with a validated uniaxial accelerometer (Lifecorder; Suzuken Co. Ltd., Nagoya, Japan) for at least 2 consecutive weeks. Detailed descriptions of the accelerometer have been published elsewhere (Kumahara et al. 2004).

Skilled dieticians assessed total energy intake, in kilocalories, with 3-day dietary records along with dietary recall interviews with each participant to elicit more information about his food intake for the 3-day period. The dietary data for each participant were analyzed using commercially available computer software (Excel Eiyo-kun, Kenpakusha, Tokyo, Japan).

\section{Exercise interventions}

All of the exercise training sessions were supervised by exercise physiologists and were conducted near our facility. The exercise program consisted primarily of $90 \mathrm{~min}$ of aerobic exercise 3 days/week for 12 weeks (36 times). Each 90 min exercise session began with 15-30 min of warm-up activities in a fitness studio and included stretching, followed by 30-60 min of fast walking and/or mild jogging performed outdoors and concluded with 15-30 min of cool-down activities. The exercise intensity was based on the percentage of the maximal heart rate (HR) attained by each participant as measured with short-range telemetry (RS400, Polar, Kempele, Finland). Briefly, the target exercise intensity was $65-80 \%$ of the age-predicted HR (220 minus age). To examine the effects of regular exercise alone and to eliminate the confounding effects of diet-induced weight loss, we advised the participants to not change their dietary habits during the study period. Energy expenditure was measured throughout the intervention in the longitudinal analysis; energy intake was assessed during weeks 9-10 of the intervention by the same method as above.

\section{Statistical analysis}

The values are expressed as the mean \pm S.D. We used Levene's test to evaluate the equality of variance and the Shapiro-Wilk test was applied to test the normality of the variables; we compared the values before and after the intervention. We used the paired Student's t-test for the parameters presenting a Gaussian distribution. When it failed to normalize the residual distributions, we used the Mann-Whitney test. The images ranged from $20 \mathrm{~cm}$ above L4-L5 $(+20 \mathrm{~cm})$ to $3 \mathrm{~cm}$ below L4-L5 $(-3 \mathrm{~cm})$, yielding 24 images per participant. Differences in the association between VAT areas measured at different sites across the abdomen and CVD risk factors were tested by comparing each age-adjusted Pearson's correlation coefficient. Pearson's correlation coefficients were also used to assess the relationship between single-slice image locations and the VAT volume. We compared each correlation coefficient with the coefficient of L4-L5 using Fisher's Z transformation method (Fisher 1915). The data were analyzed using SPSS version 13.0 for Windows package (SPSS Inc., Chicago, USA). A statistically significant level was set at $p<0.05$.

\section{Results}

\section{Baseline characteristics and changes after exercise inter- vention}

The participants' characteristics are presented in Table 1. The attendance rate was $76.4 \%$ (ranging from 41 to $100 \%$ ) for the exercise intervention. Participants significantly reduced their body weight, VAT volume, BMI and abdominal circumference during the exercise intervention. The $\dot{\mathrm{VO}_{2}}$ max was significantly increased by $10.8 \%$, and favorable changes were observed in all of the CVD risk factors. Furthermore, there was no change in the total energy intake (from $2,192 \pm 602$ to $2,161 \pm 597 \mathrm{kcal} /$ day, $p=$ 0.435 ), whereas the total energy expenditure (from 2,423 \pm 364 to $2,694 \pm 377 \mathrm{kcal} /$ day), physical activity energy expenditure (from $284 \pm 177$ to $517 \pm 183 \mathrm{kcal} /$ day) and number of steps (from $7,295 \pm 3,617$ to $11,927 \pm 3370$ steps/day) increased significantly ( $p<0.005$ for all). These improvements were mainly achieved through the supervised exercise. 
Table1. Characteristics of study participants.

\begin{tabular}{|c|c|c|c|c|}
\hline & \multirow{2}{*}{$\begin{array}{c}\text { Total participants } \\
\qquad(n=82) \\
\text { Baseline }\end{array}$} & \multicolumn{3}{|c|}{$\begin{array}{l}\text { Participants who completed intervention } \\
\qquad(n=37)\end{array}$} \\
\hline & & Baseline & Change & $\%$ Change \\
\hline Age, yr & $47.8 \pm 9.2$ & $47.3 \pm 9.7$ & - & - \\
\hline Height, cm & $170.6 \pm 6.6$ & $171.7 \pm 6.7$ & - & - \\
\hline Body weight, $\mathrm{kg}$ & $86.8 \pm 13.4$ & $88.0 \pm 14.1$ & $-1.6 \pm 3.3^{*}$ & $-1.8 \pm 3.9^{*}$ \\
\hline BMI, $\mathrm{kg} / \mathrm{m}^{2}$ & $29.8 \pm 3.8$ & $29.8 \pm 4.0$ & $-0.6 \pm 1.1^{*}$ & $-1.8 \pm 3.8^{*}$ \\
\hline Abdominal circumference, $\mathrm{cm}$ & $101.1 \pm 9.9$ & $101.9 \pm 9.4$ & $-3.7 \pm 3.0^{*}$ & $-3.4 \pm 3.0^{*}$ \\
\hline \multicolumn{5}{|l|}{ Visceral adipose tissue } \\
\hline volume, $\mathrm{cm}^{3}$ & $4,397 \pm 1,459$ & $4,134 \pm 1,452$ & $-511 \pm 555^{*}$ & $-10 \pm 12.9^{*}$ \\
\hline \multicolumn{5}{|l|}{ Subcutaneous adipose tissue } \\
\hline area (L4-L5), $\mathrm{cm}^{2}$ & $265.2 \pm 100.3$ & $259.7 \pm 99.3$ & $-16.7 \pm 31.6^{*}$ & $-6.4 \pm 13.6^{*}$ \\
\hline volume, $\mathrm{cm}^{3}$ & $4,584 \pm 1,817$ & $4,439 \pm 1,788$ & $-282 \pm 370^{*}$ & $-6.6 \pm 9.9 *$ \\
\hline $\mathrm{VO}_{2} \max , \mathrm{ml} / \mathrm{kg} / \mathrm{min}$ & $30.5 \pm 2.7$ & $33.7 \pm 4.2$ & $3.3 \pm 3.2^{*}$ & $12.9 \pm 30.5^{*}$ \\
\hline \multicolumn{5}{|l|}{ Cardiovascular disease risk factors } \\
\hline $\mathrm{TC}, \mathrm{mg} / \mathrm{dl}$ & $205.4 \pm 32.9$ & $202.6 \pm 32.6$ & $-5.4 \pm 12.4^{*}$ & $-2.5 \pm 7.3^{*}$ \\
\hline HDL-C, mg/dl & $47.9 \pm 11.8$ & $45.8 \pm 10.8$ & $5.2 \pm 5.6^{*}$ & $11.5 \pm 12.1^{*}$ \\
\hline $\mathrm{TG}, \mathrm{mg} / \mathrm{dl}$ & $175.6 \pm 129.0$ & $193.8 \pm 160.6$ & $-46.2 \pm 86.3 *$ & $-17.9 \pm 44.3^{*}$ \\
\hline $\mathrm{SBP}, \mathrm{mmHg}$ & $128.9 \pm 16.8$ & $127.5 \pm 17.8$ & $-3.7 \pm 10.0^{*}$ & $-2.3 \pm 5.9^{*}$ \\
\hline DBP, mmHg & $86.4 \pm 11.9$ & $85.1 \pm 12.2$ & $-3.3 \pm 7.7^{*}$ & $-3.7 \pm 8.0^{*}$ \\
\hline Glucose, mg/dl & $105.1 \pm 31.5$ & $101.8 \pm 27.6$ & $-7.2 \pm 25.0 *$ & $-1.8 \pm 8.6^{*}$ \\
\hline Insulin, $\mu \mathrm{U} / \mathrm{ml}$ & $10.8 \pm 6.1$ & $10.2 \pm 5.7$ & $-3.3 \pm 8.3^{*}$ & $-31.2 \pm 30.4^{*}$ \\
\hline Smoker, $\%$ & 32.1 & 25.0 & & \\
\hline Drinker, \% & 67.1 & 62.5 & & \\
\hline
\end{tabular}

BMI, body mass index; TC, total cholesterol; HDL-C, high-density lipoprotein cholesterol; TG, triglycerides; SBP, systolic blood pressure; DBP, diastolic blood pressure. Values are presented as mean \pm standard deviation. *Significant change within group by paired $t$-test $(P<0.05)$

Influence of exercise on visceral adipose tissue image areas

Table 2 shows the mean single-slice image areas at each measured level of all participants at baseline. It also shows mean image areas at baseline, post-intervention and the change in area of those who completed the exercise intervention. After the intervention, the single-slice VAT areas had decreased significantly at all measurement sites except for 19-20 cm above L4-L5. Percent change of the VAT areas had decreased significantly at most measurement sites except for 19-20 cm above and 1-3 cm below L4-L5.

\section{Single-slice image areas having the highest correlations with total VAT volume}

Fig. 1 illustrates the association between baseline VAT areas and VAT volume and the association between changes in VAT areas and VAT volume. For reference, the site marked " 0 " is the L4-L5 image, and the images collected at $1 \mathrm{~cm}$ intervals above that point (cranially) are labeled +1 to +20 , while the images collected at $1 \mathrm{~cm}$ intervals below that point (caudally) are labeled -1 to -3 . At baseline, all of the individual single-slice image areas correlated significantly with the VAT volume after adjusting for age. The correla- tion coefficients for images $2-14 \mathrm{~cm}$ above L4-L5 were significantly higher than the coefficient for the L4-L5 image. The single-slice image with the highest correlation coefficient in relation to the VAT volume was located $9 \mathrm{~cm}$ above L4-L5 $(r=0.97)$. We observed significant correlations between the individual change $(\Delta)$ in the single-slice image areas and the $\triangle \mathrm{VAT}$ volume for all of the images. The image with the highest correlation coefficient was located 8 $\mathrm{cm}$ above L4-L5 $(r=0.84)$. In addition, the images $4-9 \mathrm{~cm}$ above L4-L5 had significantly greater correlation coefficients than did L4-L5.

Associations between baseline VAT areas and CVD risk factors and between changes in both VAT areas and CVD risk factors

The associations between single-slice image VAT areas and individual CVD risk factors are shown in Table 3, with those VAT areas having significant correlations indicated by underlined $r$ values. The relationship between single-slice images and CVD risk factors at the measurement site with the highest correlations (not age adjusted) are shown in Fig. 2. There were no significant correlations between single 
Table 2. Single-slice image areas at baseline and after exercise intervention.

\begin{tabular}{|c|c|c|c|c|c|}
\hline \multirow{2}{*}{$\begin{array}{l}\text { Measurement } \\
\text { site }(\mathrm{cm})\end{array}$} & \multirow{2}{*}{$\begin{array}{l}\text { Total participants } \\
\qquad(n=82) \\
\text { Baseline }\left(\mathrm{cm}^{2}\right)\end{array}$} & \multicolumn{4}{|c|}{ Participants who completed intervention $(n=37)$} \\
\hline & & Baseline $\left(\mathrm{cm}^{2}\right)$ & After $\left(\mathrm{cm}^{2}\right)$ & Change $\left(\mathrm{cm}^{2}\right)$ & $\%$ Change \\
\hline+20 & $83 \pm 48$ & $89 \pm 53$ & $79 \pm 64$ & $-10 \pm 41$ & $-7 \pm 40$ \\
\hline+19 & $93 \pm 57$ & $102 \pm 62$ & $87 \pm 73$ & $-14 \pm 49$ & $-10 \pm 45$ \\
\hline+18 & $114 \pm 70$ & $127 \pm 76$ & $100 \pm 76$ & $-28 \pm 44^{*}$ & $-20 \pm 31 *$ \\
\hline+17 & $129 \pm 74$ & $143 \pm 81$ & $111 \pm 77$ & $-32 \pm 43^{*}$ & $-17 \pm 31 *$ \\
\hline+16 & $142 \pm 76$ & $153 \pm 82$ & $129 \pm 81$ & $-23 \pm 44^{*}$ & $-11 \pm 30 *$ \\
\hline+15 & $161 \pm 78$ & $171 \pm 83$ & $151 \pm 81$ & $-21 \pm 46^{*}$ & $-8 \pm 25^{*}$ \\
\hline+14 & $178 \pm 80$ & $190 \pm 86$ & $161 \pm 79$ & $-30 \pm 44^{*}$ & $-12 \pm 21 *$ \\
\hline+13 & $192 \pm 81$ & $203 \pm 86$ & $174 \pm 78$ & $-29 \pm 40^{*}$ & $-11 \pm 18^{*}$ \\
\hline+12 & $202 \pm 78$ & $210 \pm 82$ & $187 \pm 72$ & $-24 \pm 30^{*}$ & $-9 \pm 13^{*}$ \\
\hline+11 & $208 \pm 80$ & $217 \pm 83$ & $194 \pm 74$ & $-23 \pm 28^{*}$ & $-9 \pm 12 *$ \\
\hline+10 & $209 \pm 75$ & $218 \pm 78$ & $207 \pm 75$ & $-13 \pm 31^{*}$ & $-6 \pm 12 *$ \\
\hline+9 & $213 \pm 76$ & $222 \pm 79$ & $200 \pm 69$ & $-22 \pm 32 *$ & $-8 \pm 15^{*}$ \\
\hline+8 & $214 \pm 73$ & $219 \pm 77$ & $197 \pm 67$ & $-22 \pm 33^{*}$ & $-9 \pm 14^{*}$ \\
\hline+7 & $213 \pm 78$ & $216 \pm 81$ & $198 \pm 64$ & $-18 \pm 38^{*}$ & $-9 \pm 17 *$ \\
\hline+6 & $211 \pm 78$ & $216 \pm 79$ & $194 \pm 63$ & $-22 \pm 35^{*}$ & $-8 \pm 18^{*}$ \\
\hline+5 & $211 \pm 79$ & $216 \pm 81$ & $194 \pm 61$ & $-22 \pm 36^{*}$ & $-7 \pm 19 *$ \\
\hline+4 & $211 \pm 75$ & $215 \pm 76$ & $187 \pm 60$ & $-28 \pm 34^{*}$ & $-11 \pm 16^{*}$ \\
\hline+3 & $208 \pm 72$ & $212 \pm 73$ & $182 \pm 56$ & $-30 \pm 39^{*}$ & $-12 \pm 17 *$ \\
\hline+2 & $192 \pm 66$ & $195 \pm 67$ & $173 \pm 54$ & $-22 \pm 36^{*}$ & $-11 \pm 17^{*}$ \\
\hline+1 & $180 \pm 63$ & $184 \pm 64$ & $162 \pm 52$ & $-22 \pm 37^{*}$ & $-10 \pm 19^{*}$ \\
\hline 0 (L4-L5) & $174 \pm 64$ & $159 \pm 58$ & $138 \pm 53$ & $-13 \pm 50^{*}$ & $-9 \pm 20 *$ \\
\hline-1 & $145 \pm 50$ & $150 \pm 51$ & $136 \pm 44$ & $-14 \pm 34^{*}$ & $-6 \pm 24$ \\
\hline-2 & $133 \pm 42$ & $138 \pm 44$ & $123 \pm 40$ & $-15 \pm 33^{*}$ & $-8 \pm 26$ \\
\hline-3 & $125 \pm 37$ & $130 \pm 40$ & $119 \pm 36$ & $-11 \pm 30^{*}$ & $-6 \pm 23$ \\
\hline
\end{tabular}

VAT, visceral adipose tissue; SAT, subcutaneous adipose tissue. Values are presented as mean \pm standard deviation. *Significant change within group by paired $t$-test $(P<0.05)$. The site marked " 0 " is the L4-L5 image, and the images collected at $1 \mathrm{~cm}$ intervals above that point (cranially) are labeled +1 to +20 , while the images collected at $1 \mathrm{~cm}$ intervals below that point (caudally) are labeled -1 to -3 .

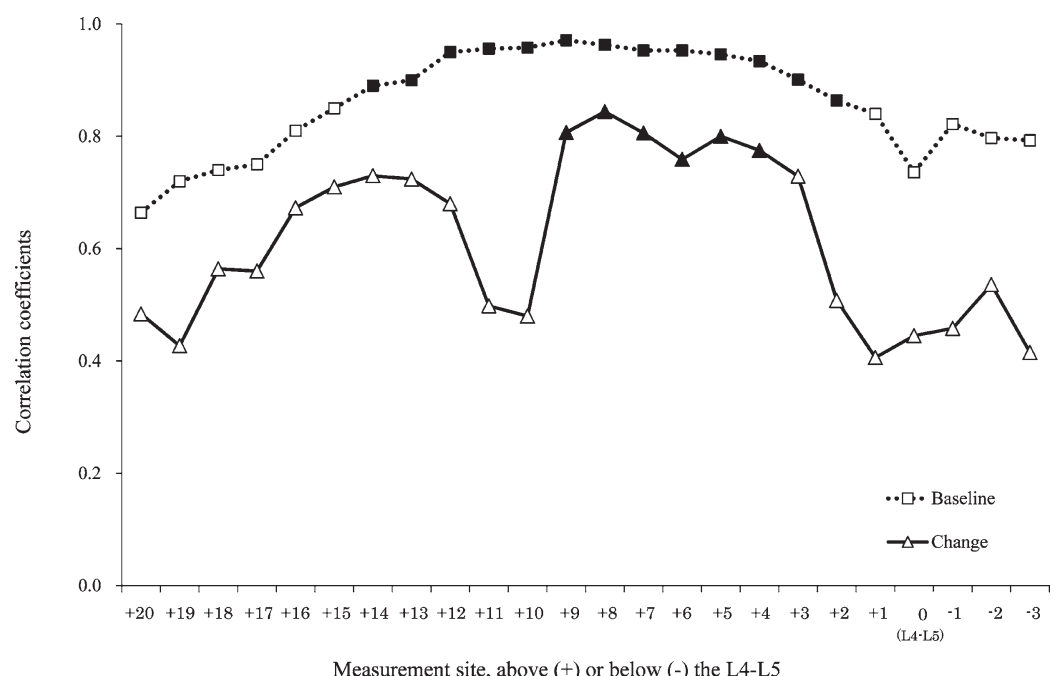

Fig. 1. Variation in the correlations between VAT volume and single-slice image areas. Baseline: $(\square)$; Change $(\triangle)$; closed symbols $(\boldsymbol{\square}$ and $\boldsymbol{\Delta}$ ) indicate correlations between VAT volume and certain measured sites that are significantly greater than between VAT volume and the standard L4-L5 measurement site. Measurement sites were from $3 \mathrm{~cm}$ below to 20 cm above L4-L5. 
Table 3. Age-adjusted partial correlations between visceral adipose tissue (VAT) areas and cardiovascular disease risk factors: change are examined between changes in VAT and changes in CVD risk factors with exercise.

\begin{tabular}{|c|c|c|c|c|c|c|c|c|c|c|c|c|}
\hline \multirow{2}{*}{$\begin{array}{l}\text { Image } \\
\text { location }\end{array}$} & \multicolumn{6}{|c|}{ Baseline $(n=82)$} & \multicolumn{6}{|c|}{ Change ( $n=37)$} \\
\hline & $\mathrm{TC}$ & HDL & TG & Glu & Insulin & SBP & $\mathrm{TC}^{\mathrm{a}}$ & HDL & $\mathrm{TG}^{\mathrm{b}}$ & Glu $^{\mathrm{b}}$ & Insulin & $\mathrm{SBP}^{\mathrm{b}}$ \\
\hline Volume & -0.31 & $-0.22 *$ & $0.24 *$ & 0.14 & 0.21 & $0.26^{*}$ & $0.38 *$ & -0.19 & $0.42 *$ & $0.39 *$ & 0.23 & 0.22 \\
\hline+20 & -0.37 & -0.20 & 0.10 & 0.08 & 0.04 & 0.06 & 0.09 & -0.29 & 0.24 & 0.14 & 0.09 & 0.10 \\
\hline+19 & -0.34 & -0.15 & 0.12 & 0.13 & 0.01 & 0.05 & 0.13 & -0.10 & 0.20 & 0.12 & 0.17 & 0.06 \\
\hline+18 & -0.25 & -0.19 & 0.12 & 0.12 & 0.03 & 0.03 & 0.16 & -0.19 & 0.25 & 0.16 & 0.31 & 0.14 \\
\hline+17 & -0.32 & -0.20 & 0.14 & 0.12 & 0.02 & 0.01 & 0.24 & -0.13 & 0.20 & 0.11 & 0.21 & 0.13 \\
\hline+16 & -0.30 & $-0.22 *$ & 0.196 & 0.14 & 0.03 & 0.01 & 0.11 & -0.24 & 0.27 & 0.20 & 0.28 & 0.19 \\
\hline+15 & -0.31 & $-0.22 *$ & $0.22 *$ & 0.16 & 0.05 & 0.03 & 0.18 & -0.27 & 0.32 & 0.24 & 0.21 & 0.12 \\
\hline+14 & -0.34 & $-0.22 *$ & $0.23 *$ & 0.17 & 0.09 & 0.04 & 0.19 & -0.31 & 0.28 & 0.23 & 0.28 & 0.08 \\
\hline+13 & -0.38 & $-0.22 *$ & $0.22 *$ & 0.13 & 0.12 & 0.06 & 0.31 & -0.42 & 0.33 & 0.27 & 0.35 & 0.06 \\
\hline+12 & -0.35 & $-0.23 *$ & $0.22 *$ & 0.15 & 0.20 & 0.03 & 0.32 & -0.33 & 0.16 & 0.16 & 0.28 & 0.03 \\
\hline+11 & -0.35 & $-0.23 *$ & $0.22 *$ & 0.17 & 0.21 & 0.01 & 0.31 & -0.24 & 0.12 & 0.24 & 0.30 & 0.04 \\
\hline+10 & -0.34 & $-0.23 *$ & $0.22 *$ & 0.14 & 0.20 & 0.02 & 0.30 & -0.11 & 0.19 & 0.25 & 0.18 & 0.11 \\
\hline+9 & -0.28 & $-0.22 *$ & $0.22 *$ & 0.10 & 0.21 & 0.07 & 0.31 & -0.05 & 0.24 & 0.18 & 0.15 & 0.16 \\
\hline+8 & -0.28 & $-0.22 *$ & $0.24 *$ & 0.08 & 0.21 & 0.11 & 0.13 & -0.13 & 0.34 & 0.20 & 0.20 & 0.06 \\
\hline+7 & -0.24 & $-0.22 *$ & $0.28 *$ & 0.10 & 0.22 & 0.10 & 0.26 & -0.04 & 0.33 & 0.23 & $0.36^{*}$ & 0.01 \\
\hline+6 & -0.33 & $-0.24 *$ & $0.29 *$ & 0.12 & $0.30 *$ & 0.11 & $0.34 *$ & -0.03 & $0.39 *$ & 0.25 & $0.37 *$ & 0.12 \\
\hline+5 & -0.34 & $-0.22 *$ & $0.26^{*}$ & 0.13 & $0.28 *$ & 0.12 & $0.42 *$ & -0.15 & $0.36^{*}$ & $0.36 *$ & 0.27 & 0.21 \\
\hline+4 & -0.37 & -0.21 & $0.26^{*}$ & 0.14 & $0.26^{*}$ & 0.12 & 0.29 & -0.26 & 0.34 & $0.32 *$ & 0.23 & 0.13 \\
\hline+3 & -0.33 & -0.18 & $0.22 *$ & 0.11 & $0.23^{*}$ & 0.12 & $0.35^{*}$ & -0.05 & 0.28 & $0.34 *$ & 0.11 & 0.15 \\
\hline+2 & -0.23 & -0.16 & 0.19 & 0.10 & $0.23^{*}$ & 0.15 & 0.28 & 0.10 & 0.08 & 0.29 & 0.12 & 0.19 \\
\hline+1 & -0.19 & -0.14 & 0.15 & 0.12 & $0.22 *$ & 0.21 & 0.22 & 0.25 & 0.17 & 0.25 & 0.14 & 0.27 \\
\hline 0 (L4-L5) & -0.18 & -0.08 & 0.10 & 0.16 & $0.22 *$ & 0.13 & $0.33^{*}$ & 0.07 & 0.12 & 0.11 & 0.13 & 0.02 \\
\hline-1 & -0.07 & -0.17 & 0.19 & 0.11 & $0.23 *$ & 0.13 & $0.34 *$ & 0.13 & 0.21 & 0.16 & 0.17 & 0.33 \\
\hline-2 & -0.09 & -0.15 & 0.16 & 0.09 & $0.25^{*}$ & 0.11 & $0.36^{*}$ & 0.07 & 0.11 & $0.32 *$ & 0.21 & 0.28 \\
\hline-3 & -0.18 & -0.18 & 0.20 & 0.10 & $0.24 *$ & 0.11 & $0.33 *$ & -0.07 & 0.15 & 0.23 & 0.28 & 0.15 \\
\hline
\end{tabular}

TC, total cholesterol; HDL, high-density lipoprotein; TG, triglyceride; Glu, glucose; SBP, systolic blood pressure. Measurement locations were from $3 \mathrm{~cm}$ below L4-L5 to $20 \mathrm{~cm}$ aboveL4-L5. The single VAT areas having the significantly correlation with TG and HDL are indicated in the table by underlined $r$ values, and the highest correlation are indicated by boldface values. *Significant change within group by paired $t$-test $(P<0.05) . \mathrm{a}=36, \mathrm{~b}=35$.

VAT areas and TC, glucose or SBP. A range of single-slice images from 5 to $15 \mathrm{~cm}$ above L4-L5 provided comparable correlations with TG and HDL-C. This optimum range of images (identified in Table 2 by the shaded region) did not include the L4-L5 image. Within the range, the highest correlation was found for the image $6 \mathrm{~cm}$ aboveL4-L5. However, insulin associated range of single-slice images from $+6 \mathrm{~cm}$ to $-3 \mathrm{~cm}$. These range included the L4-L5 image, but the strongest correlations at $6 \mathrm{~cm}$ above L4-L5.

We also examined the association between changes in various VAT areas and changes in CVD risk factors. Reductions in the single-slice image VAT areas were significantly associated with $\Delta \mathrm{TC}, \Delta \mathrm{TG}, \Delta$ glucose and $\Delta$ insulin. The $\triangle \mathrm{TC}$ correlated significantly with VAT area measurements taken at $+5-6 \mathrm{~cm}$ and -1 to $-3 \mathrm{~cm}$ relative to L4-L5; however, these correlations were not significantly greater than at L4-L5. The $\Delta$ glucose correlated only with the VAT area $5 \mathrm{~cm}$ above L4-L5, and $\triangle \mathrm{TG}$ correlated significantly only with the VAT areas 5-6 cm above L4-L5. Ainsulin also correlated only with the VAT area 6-7 $\mathrm{cm}$ above L4-L5, and these correlations were significantly greater than those with L4-L5, with the exception of $\triangle \mathrm{TG}$.

\section{Discussion}

The present study determined which measurement site is best to approximate VAT volume in cross-sectional and longitudinal studies of obese, Japanese men. We found that a single-slice image located $9 \mathrm{~cm}$ above L4-L5 explained $98 \%$ of the variance in the VAT volume at baseline, which was significantly greater than that for L4-L5 $(75 \%)$. There is a larger difference in correlation coefficients between the measurement sites in terms of change than in terms of baseline values (Fig. 1, continuous line). The change in the VAT area located $8 \mathrm{~cm}$ above L4-L5 explained $84 \%$ of the variance in the 4 VAT volume, but the change in the L4-L5 VAT area explained only $57 \%$ of the variance. This result suggests that the VAT volume estimated with a single-slice image at L4-L5 can lead to a misestimation of the accumu- 

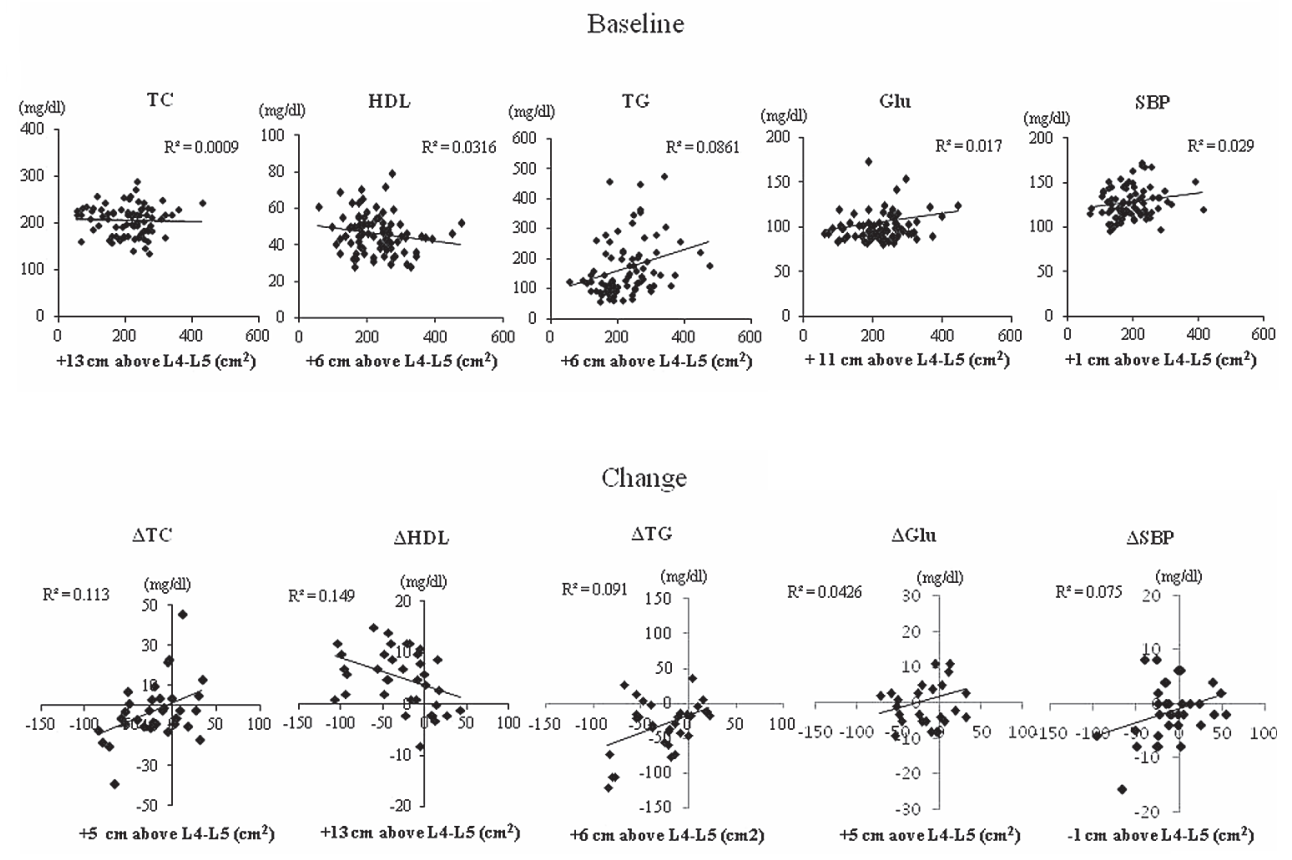

Fig. 2. Relationship between single-slice images and CVD risk factors at the measurement site having the strongest correlation to baseline VAT and change in VAT. Coefficient of determination $\left(\mathrm{R}^{2}\right)$ is analyzed by linear regression.

lation of VAT and changes that occur with exercise, which would likely lead to a misunderstanding of the effects of exercise. In agreement with the results of previous studies (Abate et al. 1997; Shen et al. 2004; Ellis et al. 2007), the measurement sites located above L4-L5 provided a substantially better estimation of the total abdominal VAT volume, compared with the VAT volume reported at L4-L5.

Another finding of this study is that the measurement sites for VAT were associated with specific CVD risk factors, and the measurement sites were also associated with a CVD risk reduction induced by the exercise intervention. There was a tendency for the VAT measurements in the mid-abdomen (5-15 cm above L4-L5) to be similarly correlated with TG and HDL-C at baseline. Shen et al. (2007) found that VAT measurement areas located $5-10 \mathrm{~cm}$ above L4-L5 have higher correlations with CVD risk factors than the VAT area at the traditional L4-L5 level after adjusting for age, race, and menopausal status. Recent findings by Irlbeck et al. (2010) also indicated that single-slice image area measurements of the VAT at L2-L3 and L3-L4 (e.g., 2-6 cm above L4-L5) had strong associations with CVD risk factors in men in a cross-sectional study. We would recommend that, if only a small number of images or a single-slice image is to be analyzed, the image should be taken $5-10 \mathrm{~cm}$ above L4-L5, rather than at L4-L5 to best evaluate CVD risk factors. In addition, according to our results, although Asians have a greater amount of VAT across the same level of BMIs and abdominal circumferences than Westerners (Kadowaki et al. 2006), the accumulation pattern of VAT may not be different among races.

In this study, we also examined whether there are dif- ferent correlations between VAT loss and CVD risk reduction after a 12-week exercise intervention. Measurements taken 5-6 $\mathrm{cm}$ above L4-L5 correlated significantly with the TC, TG, glucose and insulin levels, which is different from the findings at baseline. Although we could not support this finding, the measurement site may influence specific CVD risk factors, such as the TC, TG, glucose and insulin levels. Although our findings contrast with those of Kuk et al. (2006), who documented that the VAT measurement sites did not relate to an improved prediction for MetS, we suggest that the key differences with our results could be explained by their analytical approach. Kuk et al. (2006) used a binary logistic regression model with MetS as a dependent variable, and the measurement sites were not related to the corresponding changes in the number of MetS risk factors. Because the association with each CVD risk factor has not been considered, it may be of great importance to further investigate the associations between the VAT measurement sites and MetS and/or CVD risk factors in an interventional design.

Furthermore, Dermerath et al. (2008) reported that images located 4-8 cm cranial toL4-L5 more strongly and consistently correlate with CVD risk factors. These results should be replicated to confirm the effects of VAT patterning on CVD risk factors with more clinically relevant methods. We found that the single-slice image taken 5-6 cm above L4-L5 correlated significantly with changes in the $\mathrm{TC}, \mathrm{TG}$, glucose and insulin levels in response to the exercise intervention. This finding suggests that $5-6 \mathrm{~cm}$ above L4-L5 is a better location than L4-L5 for detecting CVD risk reduction in future clinical studies. As there were sig- 
nificant correlations only with the levels of TC, TG, glucose and insulin (not with the HDL-C level and blood pressure) in the longitudinal analysis, the respective VAT measurement sites may influence each CVD risk factor differently. Thus, the use of multiple VAT measurement sites may lead to a better prediction of CVD risk reduction in a clinical setting.

The reasons for this high correlation with CVD risk factors at the upper abdomen remain unclear. Shen et al. (2004) reported that the VAT has 2 compartments, intraperitoneal and extraperitoneal adipose tissue (IPAT and EPAT, respectively), and the IPAT is thought to be more metabolically active than the EPAT. This explanation supports the hypothesis that a single-slice image taken above L4-L5 contains more metabolically active VAT, which is consistent with the observation that the IPAT is located primarily in the upper abdomen. Moreover, several studies (Rebuffescrive et al. 1990; Bergman et al. 2006) have reported that upper abdominal VAT primarily comprises the highly lipolytic mesenteric and omental adipose tissues, which are drained by the portal vein. The upper abdominal VAT, especially around the VAT of the liver, increases the delivery of free fatty acids to the liver, which might be followed by metabolic derangements with exercise in obesity. In additions, intra-hepatic lipid accumulation could be another potential explanation. The relation between intrahepatic lipid accumulation (non-alcoholic fatty liver) and CVD has been reported by numerous population-based studies (Adams and Lindor 2007; Kolak et al. 2007). However, because we do not directly measure the VAT compartments and lipolytic activities, these mechanisms should be further investigated and replicated in studies with clinical data.

The strengths of the present study were the continuous image data obtained from $20 \mathrm{~cm}$ above to $3 \mathrm{~cm}$ below the L4-L5 area, which allowed us to ascertain the precise location of the slice having the highest correlation with CVD risk factors. In addition, the present study is the first to show associations between VAT measurement sites and both CVD risk factors and the changes in these risk factors in response to exercise in obese, Asian men. Little information on this topic is currently available, but the present study demonstrates that there are no substantial differences between races in terms of the relationship between the VAT distribution pattern and VAT volume and CVD risk factors (Shen et al. 2007; Demerath et al. 2008). Limitations of the study include only obese Japanese men and the relatively small sample of men who participated in the exercise intervention. In addition, we did not observe significant associations between VAT at L4-L5 and CVD risk factors. Although two previous studies (Shen et al. 2007; Demerath et al. 2008) also found no associations between the traditional measuring site of L4-L5 and CVD risk factors, those studies did not discuss the reason behind their results. Whereas, our data did not clarify why there was no association, a possible explanation is that our sample was relatively heterogeneous in terms of age and adiposity; future research on this topic should include subjects having a wide range of ages, BMIs and women. Therefore, our results should be applied cautiously until future studies confirm the results on larger population-based groups, as well as for other obesityrelated risks. Future studies also would need to examine the mechanisms linking adipose tissue distribution and other influencing risk factors.

\section{Conclusion}

We found that single-slice images of VAT areas located 5-6 cm above L4-L5 had the best association with CVD risk factors and with estimating the total VAT volume. The corresponding disc level 5-6 cm above L4-L5 was near L2-L3 in $87.3 \%$ of participants and precisely at L2-L3 in $13.7 \%$ of participants in our study. We also observed that a nearly identical set of VAT measurement sites had a better association with the favorable changes in CVD risk factors seen in response to regular exercise than the conventional measurement site of L4-L5. These results suggest that future studies should consider choosing a single-slice image located 5-6 cm above L4-L5 instead of (or in addition to) the L4-L5 image to examine the association with CVD risk factors.

\section{Acknowledgments}

This study was supported by the Funding Program for World-Leading Innovative R\&D on Science and Technology (First Program) and the Japan Society for the Promotion of Science (JSPS) \#23650429.

\section{Conflict of Interest}

All authors have no conflict of interest in this study.

\section{References}

Abate, N., Garg, A., Coleman, R., Grundy, S.M. \& Peshock, R.M. (1997) Prediction of total subcutaneous abdominal, intraperitoneal, and retroperitoneal adipose tissue masses in men by a single axial magnetic resonance imaging slice. Am. J. Clin. Nutr., 65, 403-408.

Adams, L.A. \& Lindor, K.D. (2007) Nonalcoholic fatty liver disease. Ann. Epidemiol., 17, 863-869.

Bergman, R.N., Kim, S.P., Catalano, K.J., Hsu, I.R., Chiu, J.D., Kabir, M., Hucking, K. \& Ader, M. (2006) Why visceral fat is bad: mechanisms of the metabolic syndrome. Obesity, 14, 16S-19S.

Burke, L.E. \& Wang, J. (2011) Treatment strategies for overweight and obesity. J. Nurs. Scholarsh., 43, 368-375.

Demerath, E.W., Reed, D., Rogers, N., Sun, S.S., Lee, M., Choh, A.C., Couch, W., Czerwinski, S.A., Chumlea, W.C., Siervogel, R.M. \& Towne, B. (2008) Visceral adiposity and its anatomical distribution as predictors of the metabolic syndrome and cardiometabolic risk factor levels. Am. J. Clin. Nutr., 88, 1263-1271.

Demerath, E.W., Shen, W., Lee, M., Choh, A.C., Czerwinski, S.A., Siervogel, R.M. \& Towne, B. (2007a) Approximation of total visceral adipose tissue with a single magnetic resonance image. Am. J. Clin. Nutr., 85, 362-368.

Demerath, E.W., Sun, S.S., Rogers, N., Lee, M., Reed, D., Choh, A.C., Couch, W., Czerwinski, S.A., Chumlea, W.C., Siervogel, R.M. \& Towne, B. (2007b) Anatomical patterning of visceral 
adipose tissue: race, sex, and age variation. Obesity, 15, 29842993.

Ellis, K.J., Grund, B., Visnegarwala, F., Thackeray, L., Miller, C.G., Chesson, C.E., El-Sadr, W. \& Carr, A. (2007) Visceral and subcutaneous adiposity measurements in adults: influence of measurement site. Obesity, 15, 1441-1447.

Fisher, R.A. (1915) Frequency distribution of the values of the correlation coefficient in samples of an indefinitely large population. Biometrika, 10, 507-521.

Fox, C.S., Massaro, J.M., Hoffmann, U., Pou, K.M., MaurovichHorval, P., Liu, C.Y., Vasan, R.S., Murabito, J.M., Meiqs, J.B., Cupples, L.A., D'Aqostino, R.B. Sr. \& O’Donnell, C.J. (2007) Abdominal visceral and subcutaneous adipose tissue compartments: association with metabolic risk factors in the Framingham Heart Study. Circulation, 116, 39-48.

Irlbeck, T., Massaro, J.M., Bamberg, F., O'Donnell, C.J., Hoffmann, U. \& Fox, C.S. (2010) Association between singleslice measurements of visceral and abdominal subcutaneous adipose tissue with volumetric measurements: the Framingham Heart Study. Int. J. Obes (Lond)., 34, 781-787.

Jakicic, J.M. (2003) Exercise in the treatment of obesity. Endocrinol. Metab. Clin. North Am., 32, 967-980.

Janssen, I., Hudson, R., Fortier, A. \& Ross, R. (2002) Effects of an energy-restrictive diet with or without exercise on abdominal fat, intermuscular fat, and metabolic risk factors in obese women. Diabetes Care, 25, 431-438.

Kadowaki, T., Sekikawa, A., Murata, K., Maegawa, H., Takamiya, T., Okamura, T., El-Saed, A., Miyamatsu, N., Edmundowicz, D., Kita, Y., Sutton-Tyrrell, K., Kuller, L.H. \& Ueshima, H. (2006) Japanese men have larger areas of visceral adipose tissue than Caucasian men in the same levels of waist circumference in a population-based study. Int. J. Obes (Lond)., 30, 1163-1165.

Keith, L.M. (1999) Abdominal cavity. In Clinically oriented ANATOMY, 4th ed., edited by Keith, L.M., Arthur, F.D. Lippincott Williams \& Wilkins, Philadelphia, PA, pp. 175-178.

Kolak, M., Westerbacka, J., Velagapudi, V.R., Waqsater, D., Yetukuri, L., Makkonen, J., Rissanen, A., Hakkinen, A.M., Lindell, M., Bergholm, R., Hamsten, A., Eriksson, P., Fisher, R.M., Oresic, M. \& Yki-Jarvinen, H. (2007) Adipose tissue inflammation and increased ceramide content characterize subjects with high liver fat content independent of obesity. Diabetes, 56, 1960-1968.

Kuk, J.L., Church, T.S., Blair, S.N. \& Ross, R. (2006) Does measurement site for visceral and abdominal subcutaneous adipose tissue alter associations with the metabolic syndrome? Diabetes Care, 29, 679-684.

Kuk, J.L., Church, T.S., Blair, S.N. \& Ross, R. (2010) Measurement site and the association between visceral and abdominal subcutaneous adipose tissue with metabolic risk in women. Obesity, 18, 1336-1340.

Kumahara, H., Schutz, Y., Ayabe, M., Yoshioka, M., Yoshitake, Y., Shindo, M., Ishii, K. \& Tanaka, H. (2004) The use of uniaxial accelerometry for the assessment of physical-activity-related energy expenditure: a validation study against whole-body indirect calorimetry. Br. J. Nutr., 91, 235-243.

Kvist, H., Chowdhury, B., Grangard, U., Tylen, U. \& Sjostrom, L. (1988) Total and visceral adipose-tissue volumes derived from measurements with computed tomography in adult men and women: predictive equations. Am. J. Clin. Nutr., 48, 1351-1361.

National Health and Nutrition Examination Survey. (2011) Ministry of Health, Labour and Welfare. http://www.mhlw.go.jp/bunya/kenkou/eiyou/dlh21-houkoku01. pdf [Accessed: June 1, 2012] (in Japanese).
Nieves, D.J., Cnop, M., Retzlaff, B., Walden, C.E., Brunzell, J.D., Knopp, R.H. \& Kahn, S.E. (2003) The atherogenic lipoprotein profile associated with obesity and insulin resistance is largely attributable to intra-abdominal fat. Diabetes, 52, 172-179.

Okura, T. \& Tanaka, K. (2000) Development of prediction equations for cardiorespiratory fitness using ratings of perceived exertion in Japanese men and women. Jpn. J. Phys. Fit. Sports Med., 48, 111-124 (in Japanese).

Physical Activity Guidelines Advisory Committee Report 2008. (2009) To the secretary of health and human services. Part A: executive summary. Nutr. Rev., 67, 114-120.

Rebuffe-scrive, M., Anderson, B., Olbe, L. \& Bjorntorp, P. (1990) Metabolism of adipose-tissue in intraabdominal depots in severely obese men and women. Metabolism, 39, 1021-1025.

Rosito, G.A., Massaro, J.M., Hoffmann, U., Ruberg, F.L., Mahabadi, A.A., Vasan, R.S., O’Donnell, C.J. \& Fox, C.S. (2008) Pericardial fat, visceral abdominal fat, cardiovascular disease risk factors, and vascular calcification in a communitybased sample: the Framingham Heart Study. Circulation, 117, 605-613.

Ross, R., Aru, J., Freeman, J., Hudson, R. \& Janssen, I. (2002) Abdominal adiposity and insulin resistance in obese men. Am. J. Physiol. Endocrinol. Metab., 282, E657-E663.

Sasai, H., Katayama, Y., Numao, S., Nakata, Y. \& Tanaka, K. (2008) Effects of exercise on visceral fat in obese middleaged men: comparison to dietary modification. Jpn. J. Phys. Fit. Sports Med., 57, 89-99 (in Japanese).

Shen, W. \& Chen, J. (2008) Application of imaging and other noninvasive techniques in determining adipose tissue mass. Methods Mol. Biol., 456, 39-54.

Shen, W., Punyanitya, M., Chen, J., Gallagher, D., Albu, J., Pi-Sunyer, X., Lewis, C.E., Grunfeld, C., Heymsfield, S.B. \& Heshka, S. (2007) Visceral adipose tissue: relationships between single slice areas at different locations and obesityrelated health risks. Int. J. Obes (Lond)., 31, 763-769.

Shen, W., Punyanitya, M., Wang, Z., Gallagher, D., St-Onge, M.P., Albu, J., Heymsfield, S.B. \& Heshka, S. (2004) Visceral adipose tissue: relations between single-slice areas and total volume. Am. J. Clin. Nutr., 80, 271-278.

Straznicky, N.E., Grima, M.T., Lambert, E.A., Eikelis, N., Dawood, T., Lambert, G.W., Nestel, P.J., Masuo, K., Sari, C.I., Chopra, R., Mariani, J.A. \& Schlaich, M.P. (2011) Exercise augments weight loss induced improvement in renal function in obese metabolic syndrome individuals. J. Hypertens., 29, 553-564.

Tanaka, K., Okura, T., Shigematsu, R., Nakata, Y., Lee, D.J., Wee, S.W. \& Yamabuki, K. (2004) Target value of intraabdominal fat area for improving coronary heart disease risk factors. Obes. Res., 12, 695-703.

Tanaka, K., Takeshima, N., Kato, T., Niihata, S. \& Ueda, K. (1990) Critical determinants of endurance performance in middleaged and elderly endurance runners with heterogeneous training habits. Eur. J. Appl. Physiol. Occup. Physiol., 59, 443-449.

The Examination Committee of Criteria for 'Obesity Disease' in Japan; Japan Society for the Study of Obesity: new criteria for 'obesity disease' in Japan. (2002) Circ. J., 66, 987-992.

The National Institute of Health and Nutrition Survey in Japan 2008, Health Service Bureau, MHLW. http://www.nih.go.jp/eiken/nns_video/fukui.wmv. [Accessed: June 1, 2012].

Yamazaki, M. \& Hayashi, T. (2002) Rapid-onset type 1 diabetes mellitus without pancreatic exocrine dysfunction. Ann. Intern. Med., 137, 145-146. 